\title{
Mode-locked laser pulse sources for wavelength division multiplexing
}

\author{
Edward Farnum ${ }^{a}$, Brandon G. Bale ${ }^{b}$ and J. Nathan Kutz ${ }^{c}$ \\ ${ }^{a}$ Department of Science, Technology and Mathematics, Kean University, Union, NJ 07083; \\ ${ }^{b}$ Photonics Research Group, Aston University, Birmingham UK B4 7ET; \\ ${ }^{c}$ Department of Applied Mathematics, University of Washington, Seattle, W A 98195-2420
}

\begin{abstract}
Recent theoretical investigations have demonstrated that the stability of mode-locked solution of multiple frequency channels depends on the degree of inhomogeneity in gain saturation. In this paper, these results are generalized to determine conditions on each of the system parameters necessary for both the stability and existence of mode-locked pulse solutions for an arbitrary number of frequency channels. In particular, we find that the parameters governing saturable intensity discrimination and gain inhomogeneity in the laser cavity also determine the position of bifurcations of solution types. These bifurcations are completely characterized in terms of these parameters. In addition to influencing the stability of mode-locked solutions, we determine a balance between cubic gain and quintic loss, which is necessary for existence of solutions as well. Furthermore, we determine the critical degree of inhomogeneous gain broadening required to support pulses in multiple frequency channels.
\end{abstract}

Keywords: mode-locked lasers, wavelength division multiplexing

\section{INTRODUCTION}

Nonlinear photonic technologies are of continued scientific interest due to their proposed performance increases over their electronic counterparts. The two most commercially successful applications of nonlinear photonics have come from two fiber optic-based applications: optical fiber communications and mode-locked fiber lasers. ${ }^{1}$ In both cases, the optical fiber serves as a nearly ideal waveguide whose dispersion and nonlinearity is used as the basis for controlling the light pulses, i.e. the optical bits. Indeed, the intensity-dependent cubic (Kerr) nonlinearity exhibited in the optical fibers has led to significant theoretical and experimental consideration of soliton solutions of the nonlinear Schrödinger equation (NLS). The robust and stable nature of the one-soliton solutions to NLS have led to their consideration as optical bits in wide range of applications far beyond optical communications and fiber lasers. Current optical fiber-communication networks increasingly rely on wavelengthdivision multiplexing (WDM) technologies in conjunction with optical time-division multiplexing (OTDM) of individual WDM channels. The combination of high-repetition-rate data streams with a large number of WDM channels has pushed transmission rates to nearly $1 \mathrm{~TB} / \mathrm{s} .{ }^{1}$ This has created a demand for all-optical transmission sources that can generate pico-second mode-locked pulses at various wavelengths. ${ }^{3-6}$ Here, we develop a lowdimensional theoretical description of the dynamics of a multiple-wavelength mode-locked laser source. We characterize the stability and interaction dynamics of the mode-locked soliton-like solutions as a function of the number of frequency channels mode-locked. Further, we develop a comprehensive theoretical treatment of the bifurcation structure, via a center manifold and normal form reduction, associated with the mode-locking behavior.

Mode-locking is a fundamentally nonlinear phenomena whereby an often perturbatively small intensitydiscrimination element in a laser cavity leads to the formation of stable and robust perturbed solitons in the laser cavity. ${ }^{7,8}$ These mode-locked pulses often behave as global attractors to the underlying laser system. It is the intensity discrimination element in the cavity that breaks the Hamiltonian and completely integrable structure of the NLS so as to achieve a globally attracting solution. In addition to intensity discrimination, amplification

Further author information: (Send correspondence to E. Ding)

J. N. Kutz: E-mail: kutz@amath.washington.edu, Telephone: 12066853029

Nonlinear Frequency Generation and Conversion: Materials, Devices, and Applications IX, edited by Peter E. Powers, Proc. of SPIE Vol. 7582, 758216 - @ 2010 SPIE

CCC code: $0277-786 \mathrm{X} / 10 / \$ 18 \cdot$ doi: $10.1117 / 12.840750$

Proc. of SPIE Vol. 7582 758216-1 
in the laser cavity must be applied in order to compensate for losses in the laser cavity. For pulsed lasers, the past decade has focused on the use of Erbium-doped fibers for amplification operating at $1550 \mathrm{~nm}$. Thus the two basic components modifying the basic NLS description is the inclusion of the cavity amplification and intensity discrimination. A wide variety of physically realizable schemes have been proposed and developed for generating the requisite intensity discrimination including nonlinear interferometry in a figure eight laser, polarization rotation in the ring laser, quantum saturable absorption in a linear cavity configuration, spectral filtering with polarization filters in a dispersion controlled cavity, and nonlinear mode-coupling in a waveguide array-based laser.

More recently, intensity discrimination methods have been used to experimentally generate mode-locked pulses at multiple frequencies simultaneously, ${ }^{3-6}$ i.e. multi-frequency mode-locking. Theoretical models of the governing equations have also been developed to characterize the resulting nonlinear interactions among the different frequency channels. ${ }^{10-13}$ These theoretical studies have primarily focused on the cubic-quintic Ginzburg-Landau model description for the mode-locking process, ${ }^{10-12}$ which is related to the master mode-locking description originally proposed by Haus. ${ }^{7,8}$ Our objective in this manuscript is to characterize the low-dimensional dynamical system description of the mode-locking process. ${ }^{12}$ Specifically, we completely characterize the underlying bifurcation structure of the mode-locking process as a function of such key critical parameters as the homogeneous and inhomogeneous gain broadening effects and intensity discrimination parameters. Our formalism and normal form reductions are capable of a complete classification of the mode-locking for two frequency operation. The methods can also be applied and extended to multiple frequencies, but the results are more difficult to extract analytically. Highlighted in the bifurcation analysis is the key role that the gain model plays in determining the stability of multiple frequency laser operation.

\section{GOVERNING EQUATIONS}

The evolution of the electromagnetic field in the laser cavity is subject to several key physical effects. In addition to the inherent effects of chromatic dispersion and self-phase modulation proposed as the basis for soliton formation and propagation, ${ }^{1,2}$ the laser cavity requires a saturating gain to counteract the net laser cavity losses incurred from output coupling and the intensity discrimination element. Thus the inclusion of chromatic dispersion, self-phase modulation, attenuation, bandwidth limited gain, and an intensity-discrimination element comprise the key components of a laser cavity. Averaging over all these physical effects that occur per round trip in the laser cavity, a master mode-locking model has been developed that uses a generic nonlinear loss to provide the necessary intensity discrimination. ${ }^{7,8}$ A wide variety of other theoretical models have also been developed to describe the mode-locking process, but here we use the cubic-quintic master modelocking equation ${ }^{7,8}$ that

has been recently demonstrated to make explicit connection to a passive laser cavity with a linear polarizer. ${ }^{14-16}$ The governing equations can be shown, via an averaging method, to result in a coupled set of partial differential equations for the electric field at each WDM frequency ${ }^{11,17}$

$$
i\left(\frac{\partial u_{n}}{\partial z}+\delta_{n} \frac{\partial u_{n}}{\partial t}\right)+\frac{1}{2} \frac{\partial^{2} u_{n}}{\partial t^{2}}+\left(1-i \beta_{n}\right)\left|u_{n}\right|^{2} u_{n}+i \gamma u_{n}-i g_{n}(z)\left(1+\tau_{n} \frac{\partial^{2}}{\partial t^{2}}\right) u_{n}+i \sigma_{n}\left|u_{n}\right|^{4} u_{n}+2 \sum_{j=1(j \neq n)}^{N}\left|u_{j}\right|^{2} u_{n}=0
$$

where $n=1,2, \cdots, N$ and $N$ is the total number of frequency channels being mode-locked. The model includes the nonlinear interaction associated with both self- and cross-phase modulation. Note that the small four-wave mixing products which appear at new sideband frequencies have been neglected. ${ }^{17,18}$ The normalizations for the amplitude $u_{n}$ arise from standard soliton scalings so that the propagation distance $z$ and time $t$ in the boosted frame are scaled in soliton units. ${ }^{10}$ Such a scaling sets the coefficient of the self-phase modulation and chromatic dispersion to unity. The energy equilibration parameters are determined from the gain bandwidth parameter $\tau_{n}$ and the linear attenuation $\gamma_{n}$. The parameter $\delta_{n}=\left(1 / v_{g_{n}}-1 / v_{g_{0}}\right)$ measures the group-velocity $\left(v_{g_{n}}\right)$ walk-off between the individual frequency channels in the boosted time frame of the center frequency (note that $v_{g_{0}}$ is the group-velocity of the center frequency). Although the group-velocity is explicitly considered, stable modelocking forms bound state solutions moving at the average group-velocity of the channels. ${ }^{11,12}$ This numerical observation is critical to the low-dimensional model developed in this manuscript. 


\subsection{Gain Modeling}

One of the most critical aspects of the multi-frequency dynamics is the gain saturation model $g_{n}(z)$. To our knowledge, no experimental studies exist that quantify the multi-frequency gain saturation behavior. However, it is known that in such a multi-frequency, broadband scenario, two gain effects are present: homogeneous gain broadening and inhomogeneous gain broadening. ${ }^{19}$ Qualitatively, the homogeneous gain broadening effect amplifies all frequencies equally. Thus in a multi-frequency scenario, all frequency channels would act to saturate the erbium optical amplifier. The inhomogeneous gain broadening amplifies in a frequency dependent manner. This implies that different frequency channels saturate independently of the entire gain spectrum of the erbium fiber. These two effects must be modeled at least qualitatively in order to achieve a physically relevant gain model. ${ }^{12}$

The qualitative gain model as in Bale, Farnum and Kutz ${ }^{12}$ incorporates both homogeneous and inhomogenous broadening effects. This simple model generalizes the standard and well-established saturable gain model of Haus. $^{7}$ Under this gain model, the degree of homogeneous and inhomogeneous gain broadening is controlled by the parameter $\alpha$, which will be treated as the primary bifurcation parameter in this manuscript. It is this parameter that ultimately determines the stability of the multi-frequency mode-locking operation. The gain in channel $n$ is given by

$$
g_{n}(z)=\alpha G_{n}(z)+(1-\alpha) G_{h}(z)
$$

with

$$
\begin{aligned}
G_{h}(z) & =\frac{2 g_{0}}{1+\left(\sum_{n=1}^{N}\left\|u_{n}\right\|^{2}\right) /\left(N e_{0}\right)}, \\
G_{n}(z) & =\frac{2 g_{0}}{1+\left\|u_{n}\right\|^{2} / e_{0}},
\end{aligned}
$$

where $\left\|u_{n}\right\|=\int_{-\infty}^{\infty}\left|u_{n}\right|^{2} d t, e_{0}$ is the saturation energy of the cavity, and $g_{0}$ measures the strength of the gain pumping. Here $G_{h}\left(G_{n}\right)$ models the homogeneously (inhomogeneously) saturated gain in channel $n$. The inhomogeneously broadened gain is often called the self-saturation. Note that the total gain $g_{n}(z)$ models both homogeneous and inhomogenous gain broadening as a linear combination between the two effects. The parameter $\alpha$ measures the strength of each relative to each other. Indeed, $\alpha$ acts as a homotopy parameter where $\alpha=0$ gives purely homogeneous gain broadening and $\alpha=1$ generates only inhomogeneous (self-saturating) gain broadening.

Variations in the gain saturation homogeneity in erbium-doped fiber amplifiers is well known to be influenced by a number of factors, including temperature. ${ }^{19}$ Thus given the absence of a comprehensive experimental study of the degree of homogeneous to inhomogeneous gain broadening, it is difficult to estimate the parameter $\alpha$. However, provided there is only a small amount of inhomogeneous gain broadening, it will be shown that the laser cavity can support stable multi-frequency operation. This is in agreement with the effort to use enhanced inhomogeneous gain broadening for gain equilization in WDM systems. ${ }^{19}$ As with WDM systems, the multifrequency mode-locking stability is greatly influenced by enhancing the inhomogenous gain broadening.

\subsection{Low-dimensional (Reduced) Model}

The governing equation (1) is a partial differential equation modeling the spatial-temporal evolution of electromagnetic energy in the laser cavity. To obtain analytic insight into the dynamics of this model, a variational method can be used to describe the complete evolution problem with ordinary differential equations that govern the evolution of a finite set of pulse parameters. The literature regarding variational reductions in nonlinear Schrödinger systems is vast, ${ }^{20}$ and has been used to describe various aspects of mode-locking behavior ${ }^{21-27}$ as well as general Ginzburg-Landau systems. ${ }^{28}$ The variational method is traditionally rooted in the Hamiltonian nature of the system, i.e. it is assumed that some conserved energy functional can be constructed. Classical Hamiltonian theory then allows for the construction of the associated Lagrangian via a Legendre transformation. The variational reduction then applies the Euler-Lagrange equations to the free parameters in the ansatz 
assumption. A modified variational reduction ${ }^{29}$ is necessary since (1) contains dissipative terms due to gain saturation and intensity discrimination. ${ }^{12}$ Assuming an ansatz of the form

$$
\begin{aligned}
u_{n}(z, t) & =\eta_{n}(z) \operatorname{sech}\left(\omega_{n}(z) t\right) \exp \left[i \phi_{n}(z)\right] \\
\omega_{n}(z) & =\left(\eta_{n}^{2}(z)+2 \sum_{j=1(j \neq n)}^{N} \eta_{j}^{2}(z)\right)^{1 / 2}
\end{aligned}
$$

is motivated by the exact solutions of (1) with $\sigma=0 .{ }^{11}$ Further, simulations of (1) suggest that pulses at different frequencies lock to the same group-velocity so that no center position variable evolution is assumed in the ansatz. ${ }^{11,12}$ This gives the coupled amplitude evolution equations

$$
\begin{aligned}
\frac{d \eta_{n}}{d z} & =\frac{\eta_{n}}{6}\left(g_{n}\left(3-\tau_{n} \omega_{n}^{2}\right)-3 \gamma+2 \beta_{n} \eta_{n}^{2}-\frac{8}{5} \sigma_{n} \eta_{n}^{4}\right) \\
g_{n} & =\frac{2 g_{0}\left(1+\left(1-\alpha\left(\frac{N-1}{N}\right)\right) 2 Y_{n}+\frac{\alpha}{N} \sum_{j=1(j \neq n)}^{N} 2 Y_{j}\right)}{\left(1+2 y_{n}\right)\left(N+2 Y_{n}+\sum_{j=1(j \neq n)}^{N} 2 Y_{j}\right) / N}
\end{aligned}
$$

where the substitution $Y_{j}=\eta_{j}^{2} / \omega_{j}, Y_{n}=\eta_{n}^{2} / \omega_{n}$ is made for clarity. The ansatz chosen for our variational ansatz is unchirped, in contrast to the exact solutions to Hauss master mode-locking equation, which in general have a non-trivial phase profile. However, in the anomalous dispersion regime, the magnitude of this phase chirp is known to be small. For example, with the system parameters we used, the phase chirp for solutions to the full simulations are on the order of $\mathrm{O}\left(10^{-3}\right)$ for the single channel case, and $\mathrm{O}\left(10^{-2}\right)$ for the dual-channel case. Furthermore, those hyperbolic secant solutions provide only a guide for choosing an ansatz, since such solutions are not generally admissible for the case when sigma is not equal to zero. Given the small size of the chirp in comparison to the other system parameters, we decided to neglect it in our ansatz. If, on the other hand, we had been modeling pulses in the normal dispersion, then neglecting the chirp would certainly be inappropriate. Moreover, our recent findings show the variational reduction to be quite accurate in depicting the dynamics. ${ }^{12}$

It is important to note that since only trivial phase profiles are considered in the ansatz (4), the cross-phase modulation, which exchanges nonlinear phase across different frequency channels, does not explicitly account for amplitude coupling. In this model, the direct coupling between neighboring channels largely occurs due to the homogeneous gain broadening effects in (5). The analysis in the remainder of the manuscript concerns the coupled amplitude equations (5) in both the dual- and $N$-frequency system. Note that unless stated otherwise, the following parameter values are taken in all simulations: $\beta_{n}=0.05, \sigma_{n}=0.01, \tau=0.10, g_{0}=0.25$, and $\gamma=0.2167$.

\section{DUAL FREQUENCY OPERATION}

In this section we consider dual-frequency operation with identical equation parameters at each frequency, i.e. $\beta_{1}=\beta_{2}, \tau_{1}=\tau_{2}$, etc. Non-identical parameters can be considered, however the complexity of the system restricts analytical progress. ${ }^{12}$ For the reduced model (5), the dynamics simplifies to the coupled ordinary differential equations

$$
\begin{aligned}
& \frac{d \eta_{1}}{d z}=\frac{\eta_{1}}{6}\left(g_{1}\left(3-\tau \omega_{1}^{2}\right)-3 \gamma+2 \beta \eta_{1}^{2}-\frac{8}{5} \sigma \eta_{1}^{4}\right)=f_{1}\left(\eta_{1}, \eta_{2}\right) \\
& \frac{d \eta_{2}}{d z}=\frac{\eta_{2}}{6}\left(g_{2}\left(3-\tau \omega_{2}^{2}\right)-3 \gamma+2 \beta \eta_{2}^{2}-\frac{8}{5} \sigma \eta_{2}^{4}\right)=f_{2}\left(\eta_{1}, \eta_{2}\right)
\end{aligned}
$$

with the gain given by

$$
\begin{aligned}
& g_{1}=\frac{2 g_{0}\left(1+(2-\alpha) \eta_{1}^{2} / \omega_{1}+\alpha \eta_{2}^{2} / \omega_{2}\right)}{\left(1+2 \eta_{1}^{2} / \omega_{1}\right)\left(1+\eta_{1}^{2} / \omega_{1}+\eta_{2}^{2} / \omega_{2}\right)} \\
& g_{2}=\frac{2 g_{0}\left(1+(2-\alpha) \eta_{2}^{2} / \omega_{2}+\alpha \eta_{1}^{2} / \omega_{1}\right)}{\left(1+2 \eta_{2}^{2} / \omega_{2}\right)\left(1+\eta_{2}^{2} / \omega_{2}+\eta_{1}^{2} / \omega_{1}\right)} .
\end{aligned}
$$


Dual-frequency operation presents a simplified model that allows for the visualization of the overall dynamics in a two-dimensional phase space. ${ }^{31}$ Further, consideration of dual-frequency operation provides key insights for the general $N$-frequency operation.

\subsection{Equilibrium Solutions}

Equilibrium solutions can be found by setting the right-hand side of (6) to zero. Here we only consider the fixed points in the first quadrant where $\eta_{1}>0, \eta_{2}>0$. Fixed points in other quadrants exist and represent solutions that are out of phase. However, (6) is invariant under the transformation $\eta_{n} \rightarrow-\eta_{n}$, thus it suffices to only consider solutions where $\eta_{1}>0, \eta_{2}>0$.

There exists an equilibrium solution $P_{1}=\left(\eta_{1}, \eta_{2}\right)=(\hat{\eta}, \hat{\eta})$ where the amplitude in each channel is identical. This corresponds to the ideal case where equal mode-locked pulsed solutions at each frequency are achieved. For this equilibrium solution, the gain (7) is independent of the choice of gain model (independent of $\alpha$ ), and is given by

$$
g_{1}=g_{2}=\frac{2 g_{0}}{1+(2 / \sqrt{3}) \hat{\eta}} .
$$

Substituting $P_{1}$ and the gain values into $f_{1}$ and $f_{2}$ and setting it to zero we obtain the quintic polynomial

$$
\begin{aligned}
F(\hat{\eta})=\left(6 g_{0}-3 \gamma\right) & -2 \sqrt{3} \gamma \hat{\eta}+\left(2 \beta-6 g_{0} \tau\right) \hat{\eta}^{2} \\
& +\frac{4}{\sqrt{3}} \beta \hat{\eta}^{3}-\frac{8}{5} \sigma \hat{\eta}^{4}-\frac{16}{5 \sqrt{3}} \sigma \hat{\eta}^{5}=0
\end{aligned}
$$

which gives the solution (numerically) for $\hat{\eta}$. For the physically reasonable system parameters outlined above, $F(\hat{\eta})$ has one real root at $\hat{\eta}=1.1966$, which is in agreement with the observed fixed point in the phase plane analysis. ${ }^{12}$

A second class of fixed points lie on the $\eta_{1}$ and $\eta_{2}$ axes. The fixed points $P_{2}=(\hat{\eta}, 0)$ and $P_{3}=(0, \hat{\eta})$ correspond to solutions in which a single channel dominates while the field in the other channel is zero. Again it is sufficient to find the roots of a single polynomial

$$
\begin{gathered}
G(\hat{\eta}, \alpha)=\left(6 g_{0}-3 \gamma\right)+\left(6 g_{0}(2-\alpha)-9 \gamma\right) \hat{\eta}+\left(2 \beta-2 g_{0} \tau-6 \gamma\right) \hat{\eta}^{2} \\
\quad+\left(6 \beta-2 g_{0} \tau(2-\alpha)\right) \hat{\eta}^{3}+\left(4 \beta-\frac{8}{5} \sigma\right) \hat{\eta}^{4}-\frac{24}{5} \sigma \hat{\eta}^{5}-\frac{16}{5} \sigma \hat{\eta}^{6} .
\end{gathered}
$$

to obtain values of $\hat{\eta}$. Note that for $P_{2}$ and $P_{3}$ the position of the roots depends on the inhomogeneity parameter $\alpha$. Figure 1 shows the value of the real root of (10) as a function of $\alpha$. It is clear that as the level of inhomogeneity is increased, the amplitude decreases.

Finally, a third class of equilibrium solutions exists for a restricted interval of the inhomogeneity parameter $\alpha .{ }^{12}$ This represents dual-frequency operation with mode-locked pulse solutions that have different amplitudes and widths in each channel. Thus the (symmetric) fixed points are given by $P_{4}=(\hat{\eta}, \bar{\eta})$ and $P_{5}=(\bar{\eta}, \hat{\eta})$, where $\hat{\eta} \geq \bar{\eta}$. The values $\hat{\eta}$ and $\bar{\eta}$ can be found by setting the right-hand side of (6) to zero and solving the resulting two coupled polynomials numerically. It is important to note that there is a restricted $\alpha$ interval in which real values for $P_{4}$ and $P_{5}$ can be found. In particular, at the bottom of this interval the fixed point $P_{4}$ coalesces with the fixed point $P_{2}$, and at the top of this interval $P_{4}$ merges with $P_{1}$. Similarly, at the bottom of this interval the fixed point $P_{5}$ coalesces with the fixed point $P_{3}$, and at the top of this interval $P_{5}$ merges with $P_{1} .{ }^{12}$ The exact interval values can be found by the linear stability analysis that follows.

\subsection{Linear Stability Analysis}

The linear stability of the fixed points can be found by examining the eigenvalues of the Jacobian matrix evaluated at each fixed point ${ }^{31}$

$$
J\left(\eta_{1}, \eta_{2}\right)=\left[\begin{array}{ll}
A\left(\eta_{1}, \eta_{2}\right) & B\left(\eta_{1}, \eta_{2}\right) \\
C\left(\eta_{1}, \eta_{2}\right) & D\left(\eta_{1}, \eta_{2}\right)
\end{array}\right]
$$




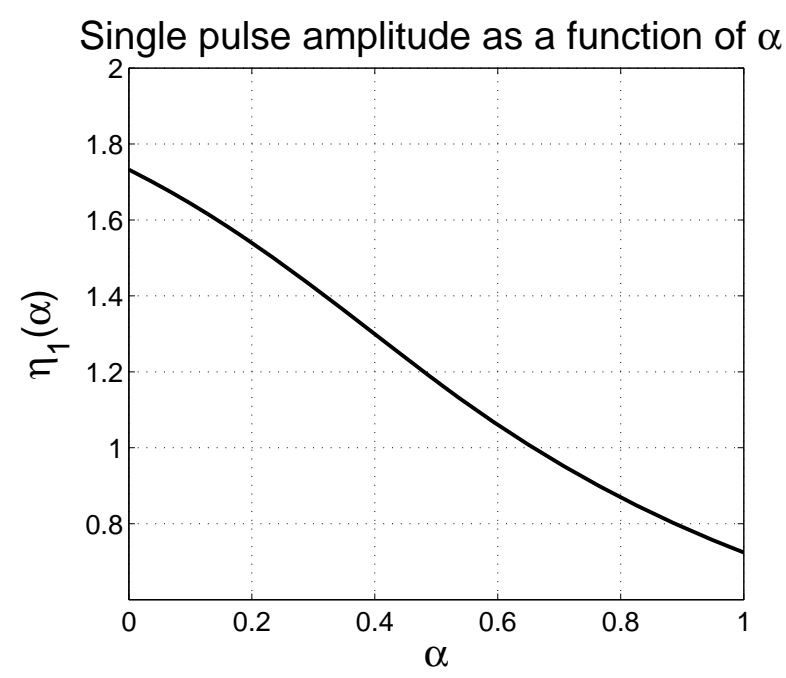

Figure 1 . In contrast to the $\eta_{1}=\eta_{2}$ case, the position of the $\left(\eta_{1}, 0\right)$ solution depends on the value of gain inhomogeneity $\alpha$. In the single pulse operation, changing $\alpha$ determines the amount of gain an individual channel receives.

where

$$
\begin{aligned}
A & =\frac{1}{6}\left[\left(3-\tau\left(3 \eta_{1}^{2}+2 \eta_{2}^{2}\right)\right) g_{1}\right. \\
& \left.+\left(3-\tau\left(\eta_{1}^{2}+2 \eta_{2}^{2}\right)\right) \eta_{1} \frac{\partial g_{1}}{\partial \eta_{1}}-3 \gamma+6 \beta \eta_{1}^{2}-8 \sigma \eta_{1}^{4}\right] \\
B & =\frac{\eta_{1}}{6}\left[-4 \tau \eta_{2} g_{1}+\left(3-\tau\left(\eta_{1}^{2}+2 \eta_{2}^{2}\right)\right) \frac{\partial g_{1}}{\partial \eta_{2}}\right] \\
C & =\frac{\eta_{2}}{6}\left[-4 \tau \eta_{1} g_{2}+\left(3-\tau\left(2 \eta_{1}^{2}+\eta_{2}^{2}\right)\right) \frac{\partial g_{2}}{\partial \eta_{1}}\right] \\
D & =\frac{1}{6}\left[\left(3-\tau\left(2 \eta_{1}^{2}+3 \eta_{2}^{2}\right)\right) g_{2}\right. \\
& \left.+\left(3-\tau\left(2 \eta_{1}^{2}+\eta_{2}^{2}\right)\right) \eta_{2} \frac{\partial g_{2}}{\partial \eta_{2}}-3 \gamma+6 \beta \eta_{2}^{2}-8 \sigma \eta_{2}^{4}\right] .
\end{aligned}
$$

We first consider the linear stability at the equal amplitude equilibrium solution $P_{1}=(\hat{\eta}, \hat{\eta})$. Since the amplitudes and gain values are equal $\left(g_{1}=g_{2}=g\right)$, it is easy to see from (12) that the diagonal entries of $J$ are equal $(A(\hat{\eta}, \hat{\eta})=D(\hat{\eta}, \hat{\eta}))$, as well as the off-diagonal entries $(B(\hat{\eta}, \hat{\eta})=C(\hat{\eta}, \hat{\eta}))$. Using this symmetry, the eigenvalues are given by

$$
\begin{aligned}
& \lambda_{1}=\frac{1}{6}\left[3\left(1-3 \tau \hat{\eta}^{2}\right) g-\frac{6 g}{\sqrt{3}} \frac{1-\tau \hat{\eta}^{2}}{\left(1+\frac{2}{\sqrt{3}} \hat{\eta}\right)} \hat{\eta}-3 \gamma+6 \beta \hat{\eta}^{2}-8 \sigma \hat{\eta}^{4}\right] \\
& \lambda_{2}=\frac{1}{6}\left[\left(3-\tau \hat{\eta}^{2}\right) g-\alpha \frac{14 g\left(1-\tau \hat{\eta}^{2}\right)}{\sqrt{3}\left(1+\frac{2}{\sqrt{3}} \hat{\eta}\right)} \hat{\eta}-3 \gamma+6 \beta \hat{\eta}^{2}-8 \sigma \hat{\eta}^{4}\right] .
\end{aligned}
$$

Note that $\lambda_{1}<0$ is independent of the gain model (independent of $\alpha$ ). There is a critical value of $\alpha=\alpha_{c}$ where the eigenvalue $\lambda_{2}=0$. Setting (13b) to zero gives

$$
\alpha_{c}=-(\sqrt{3}+2 \hat{\eta}) \frac{\left(3 \gamma-6 \beta \hat{\eta}^{2}+8 \sigma \hat{\eta}^{4}-\left(3-\tau \hat{\eta}^{2}\right) g\right)}{14 \eta g\left(1-\tau \hat{\eta}^{2}\right)} \approx 0.2947 .
$$

For values $\alpha<\alpha_{c}$, the equilibrium point $P_{1}=(\hat{\eta}, \hat{\eta})$ is unstable since $\lambda_{2}>0$. For $\alpha=\alpha_{c}$, $P_{1}$ changes its stability and becomes a stable node for $\alpha>\alpha_{c}$. A detailed bifurcation analysis of this fixed point as a function 


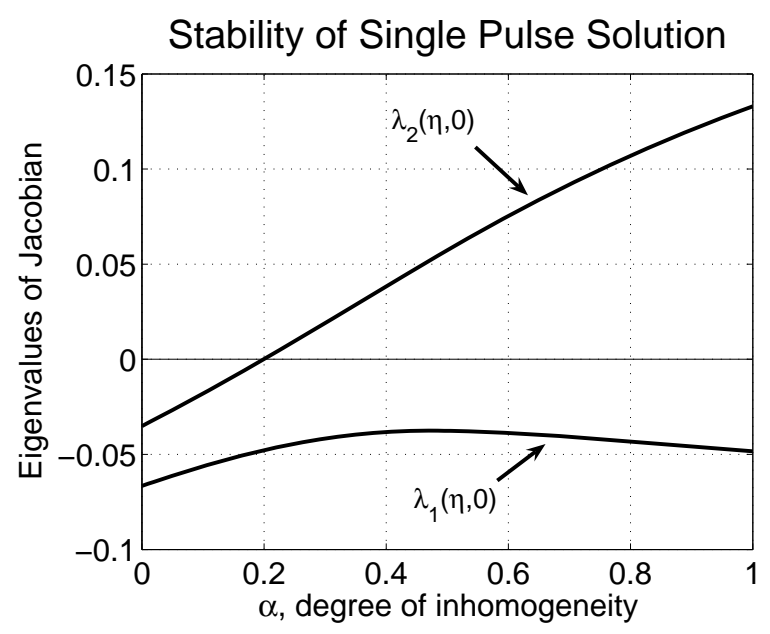

Figure 2. The stability of the $\left(\eta_{1}, 0\right)$ solution depends only on the sign of $\frac{\partial f_{2}}{\partial \eta_{2}}$ evaluated at $\left(\eta_{1}, 0\right)$. Note that the position of $\eta_{1}$ depends on the choice of $\alpha$. The figure shows that the single pulse solution is stable for $\alpha<0.1997$

of $\alpha$ is considered in the next section. The linear stability analysis of the fixed point $P_{1}$ shows that to achieve dual-frequency operation, a sufficient amount of inhomogeneous gain broadening is required. This is consistent with numerical simulations ${ }^{11,12}$ and efforts to enhance inhomogeneous gain broadening in WDM system. ${ }^{19}$

The fixed points $P_{2}=(\hat{\eta}, 0)$ and $P_{3}=(0, \hat{\eta})$ represent single-channel operation. Due to the symmetry of $(6)$, the linear stability analysis for $P_{2}$ is the same as for $P_{3}$, thus allowing us to only consider $P_{2}$. Evaluating the Jacobian (11) at the fixed point $P_{2}$ we see that $B(\hat{\eta}, 0)=C(\hat{\eta}, 0)=0$, resulting in the eigenvalues

$$
\lambda_{1}=A(\hat{\eta}, 0), \quad \lambda_{2}=D(\hat{\eta}, 0) .
$$

Since the position of $\hat{\eta}$ depends on the value of $\alpha$, characterizing the stability as a function of the gain broadening parameter is difficult. Figure 2 shows the numerical calculation of the eigenvalues $A$ and $D$ at the fixed point $P_{2}$ as a function of $\alpha$. The eigenvalue associated with $A$ is always negative, where at $\alpha=\alpha_{s}=0.1997$ the eigenvalue associated with $D$ becomes positive. Thus a bifurcation occurs at $\alpha_{s}$ where the stable fixed point at $P_{2}$ goes unstable for all $\alpha>\alpha_{s}$. Further, it can be shown for $\alpha$ values just above $\alpha_{s}$, two new stable nodes exist on either side of the fixed point $P_{2}$. Thus this instability can be described by a standard pichfork bifurcation. ${ }^{31}$

The same pitchfork bifurcation occurs at $P_{3}=(0, \hat{\eta})$ at $\alpha=\alpha_{s}$. Thus for $\alpha$ slightly greater than $\alpha_{s}$ two new stable fixed points exist $\left(P_{4}\right.$ and $\left.P_{5}\right)$ in the first quadrant so that there are five fixed points in total. These new equilibrium points represent dual frequency operation where the mode-locked pulses have different amplitudes as denoted by $P_{4}=(\hat{\eta}, \bar{\eta})$ and $P_{5}=(\bar{\eta}, \hat{\eta})$ in the previous subsection. A linear stability analysis of $P_{4}$ and $P_{5}$ can be performed numerically and reveals that these solutions are always stable nodes. However, as discussed in the previous section these solutions exist for a restricted $\alpha$ interval. Indeed, the exact interval has been found from the $\alpha$ values at the corresponding bifurcations of the fixed points $P_{1}, P_{2}$ and $P_{3}$. Thus the stable equilibrium solutions $P_{4}$ and $P_{5}$ exist from $\alpha_{s}=0.1997<\alpha<\alpha_{c}=0.2947$ for the physically reasonable values considered.

Table 1 summarizes the equilibrium solutions and their linear stability for the reduced model (6). It is clear that by increasing the amount of inhomogeneous gain broadening, stable dual-frequency operation can be achieved. Indeed, it is the inhomogeneous gain broadening parameter $\alpha$ and the bifurcations of the fixed points that effectively controls dual-frequency operation. In the following section a detailed bifurcation analysis is given.

\section{MULTI FREQUENCY $(N>2)$ MODE-LOCKING}

As the number of frequency channels is increased, there are numerous fixed points to the system (5). ${ }^{12}$ Here we are concerned with the stability of the equilibrium solution $P_{1}=\left(\eta_{1}, \eta_{2}, \cdots, \eta_{N}\right)=(\hat{\eta}, \hat{\eta}, \cdots, \hat{\eta})$ where the amplitude in each channel is identical. This corresponds to the ideal case where $N$ equal mode-locked pulsed solutions at each frequency are achieved. 


\begin{tabular}{|l|c|c|r|}
\hline$\left(\eta_{1}, \eta_{2}\right)$ & $0 \leq \alpha<\alpha_{s}$ & $\alpha_{s} \leq \alpha<\alpha_{c}$ & $\alpha \geq \alpha_{c}$ \\
\hline \hline$P_{1}=(\hat{\eta}, \hat{\eta})$ & unstable & unstable & stable \\
\hline$P_{2}=(\hat{\eta}, 0)$ & stable & unstable & unstable \\
\hline$P_{3}=(0, \hat{\eta})$ & stable & unstable & unstable \\
\hline$P_{4}=(\hat{\eta}, \bar{\eta})$ & N.A. & stable & N.A. \\
\hline$P_{5}=(\bar{\eta}, \hat{\eta})$ & N.A. & stable & N.A. \\
\hline
\end{tabular}

Table 1. Summary of the fixed points and their linear stability for dual-frequency mode-locking in the reduced model (6). The solutions and their stability depend on the degree of inhomogeneous gain broadening parameter $\alpha$. Note that for the specific parameters considered, $\alpha_{s}=0.1997$ and $\alpha_{c}=0.2947$.

Similar to dual-frequency operation, the gain $(5 \mathrm{~b})$ is independent of the choice of gain model (independent of $\alpha$ ), and is given by

$$
g_{1}=g_{2}=\cdots=g_{N} \equiv g=\frac{2 g_{0}}{\left(1+\frac{2}{\sqrt{2 N-1}} \hat{\eta}\right)} .
$$

The fixed point amplitude $\hat{\eta}$ can be found by setting (5a) equal to zero and solving the quintic polynomial

$$
\begin{aligned}
F_{N}=3\left(2 g_{0}-\gamma\right) & -\frac{6 \gamma}{\sqrt{2 N-1}} \hat{\eta}+2\left(\beta-(2 N-1) g_{0} \tau\right) \hat{\eta}^{2} \\
& +\frac{4 \beta}{\sqrt{2 N-1}} \hat{\eta}^{3}-\frac{8 \sigma}{5} \hat{\eta}^{4}-\frac{16 \sigma}{5 \sqrt{2 N-1}} \hat{\eta}^{5}
\end{aligned}
$$

The linear stability of the solution $P_{1}$ can easily be calculated due to the symmetry of both the fixed point $P_{1}$ and the gain equation (5b). The Jacobian $J(\hat{\eta})$ is an $N \times N$ constant matrix given by

$$
6 J_{i j}=\left\{\begin{array}{cc}
\left(3-\tau(2 N-1) \hat{\eta}^{2}\right) g+\left.\left(3-\tau(2 N+1) \hat{\eta}^{2}\right) \hat{\eta} \frac{\partial g_{i}}{\partial \eta_{i}}\right|_{P_{1}} & \\
-3 \gamma+6 \beta \hat{\eta}^{2}-8 \sigma \hat{\eta}^{4} & \text { if } i=j \\
{\left[-4 \tau \hat{\eta} g+\left.\left(3-\tau(2 N-1) \hat{\eta}^{2}\right) \frac{\partial g_{i}}{\partial \eta_{j}}\right|_{P_{1}}\right] \hat{\eta}} & \text { if } i \neq j .
\end{array}\right.
$$

Note that due to the symmetry of the gain (5b), the derivatives in the diagonal elements $\partial g_{i} /\left.\partial \eta_{i}\right|_{P_{1}}$ are equal for all $i=1 \cdots N$. Similarly, the derivatives $\partial g_{i} / \partial \eta_{j}$ in the off-diagnal elements $(i \neq j)$ are equivalent at the fixed point $P_{1}$. Since the Jacobian matrix is circulant with off-diagonal elements of equal value, there are only two distinct eigenvalues

$$
\begin{aligned}
\lambda_{1} & =A-B \\
\lambda_{2} & =A+(N-1) B
\end{aligned}
$$

where $A$ denotes the diagonal elements in (18) and $B$ the off-diagonal elements. Note that $\lambda_{1}$ has algebraic multiplicity $N-1$. Using (18) in (19) the eigenvalues are given by

$$
\lambda_{j}=\frac{1}{6}\left[A_{j}(N ; \hat{\eta})+B_{j}(N ; \hat{\eta})\left(C_{j}(N)+\alpha D_{j}(N)\right)\right]
$$




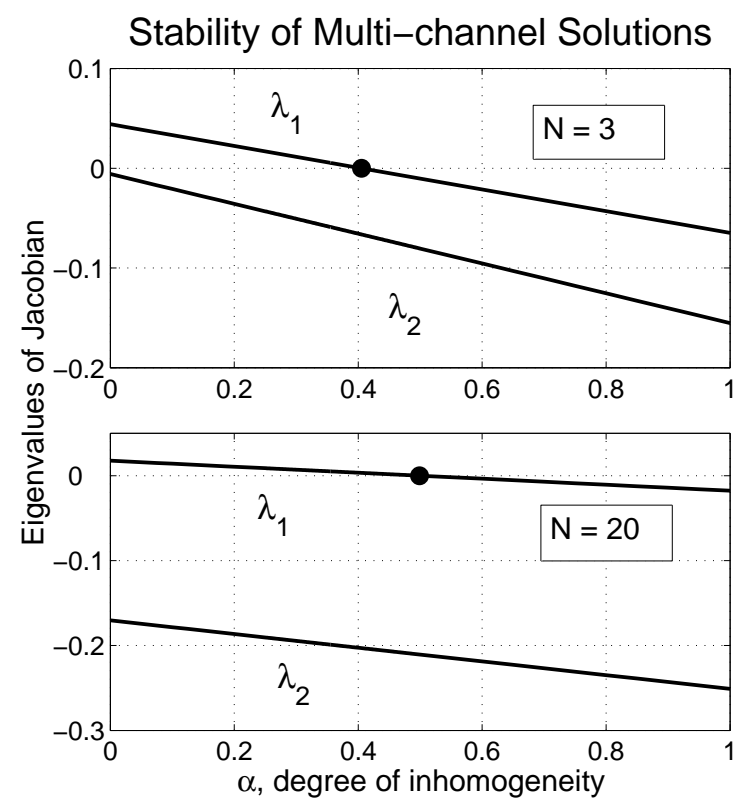

Figure 3. For multifrequency operation, the Jacobian matrix has two distinct eigenvalues. The first eigenvalue is negative for sufficient gain inhomogeneity parameter $\alpha$. The second eigenvalue is always negative. Stable multi-pulse operation can be achieved when all eigenvalues are negative. Shown above are the eigenvalues for three channel operation and twenty channel operation.

where

$$
\begin{aligned}
A_{1}(N ; \hat{\eta}) & =\left(3-\tau(2 N-3) \hat{\eta}^{2}\right) g-3 \gamma+6 \beta \hat{\eta}^{2}-8 \sigma \hat{\eta}^{4} \\
A_{2}(N ; \hat{\eta}) & =\left(3-\tau(6 N-3) \hat{\eta}^{2}\right) g-3 \gamma+6 \beta \hat{\eta}^{2}-8 \sigma \hat{\eta}^{4} \\
B_{1}=B_{2} & =\frac{\left.3-\tau(2 N-1) \hat{\eta}^{2}\right) \hat{\eta} g}{N(2 N-1)^{\frac{3}{2}}(1+2 / \sqrt{2 N-1} \hat{\eta})} \\
C_{1}(N) & =4 N-8 \\
C_{2}(N) & =8 N^{2}-22 N+12 \\
D_{1}(N) & =-8 N^{2}-2 N+8 \\
D_{2}(N) & =-20 N^{2}+32 N-12
\end{aligned}
$$

Figure (3) shows the eigenvalues for the case of $N=3$ (top) and $N=20$ (bottom) as a function of the gain ratio parameter $\alpha$. Note that for both scenarios, $\lambda_{2}<0$ for all $\alpha \leq 1$. Further, $\lambda_{1}>0$ for $0 \leq \alpha<\alpha_{c}$. Here $\alpha_{c}$ determines the critical amount of inhomogeneous gain broadening for which stable equal-pulse multi-frequency mode-locking is achieved. The trends of the eigenvalues depicted in Fig. (3) are consistent for all $N>2$. Indeed, $\lambda_{2}$ is always negative whereas $\lambda_{1}$ goes from positive to negative at some critical value $\alpha_{c}$. We can find the dependence of $\alpha_{c}$ on $N$ by setting $\lambda_{1}$ to zero and solving for $\alpha$, giving

$$
\alpha_{c}=-\frac{1}{D_{1}(N)}\left[\frac{A_{1}(N ; \hat{\eta})}{B_{1}(N ; \hat{\eta})}+C_{1}(N)\right]
$$

This result gives the necessary ratio of inhomogeneous gain broadening needed to achieve equal-pulse $N$-frequency mode-locking. An important result of (22) is the amount of inhomogeneous gain broadening necessary for stable equal-pulse multi-frequency mode-locking for any number of frequency channels. Figure(4) shows the critical value of $\alpha$ necessary to stabilize equal-pulse multi-frequency mode-locking as a function of $N$. We see that $\alpha_{c}(N)$ increases with $N$, and asymptotically approaches 0.5 for large values of $N$. Thus if half of the total gain 


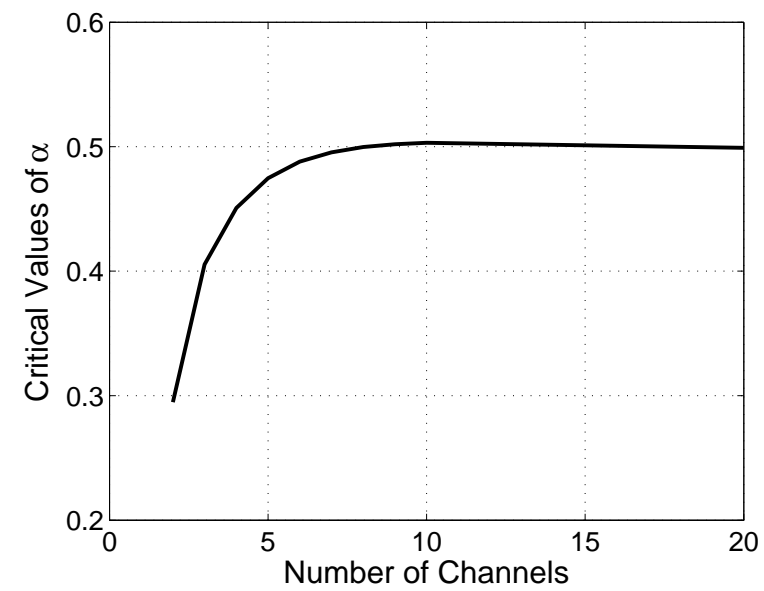

Figure 4. The critical amount of inomogenous broadening $\left(\alpha_{c}\right)$ required for multi-pulse operation depends on the number of frequency channels $N$. As $N$ becomes large, this critical value tends asymptotically to 0.5 , indicating that stable multi-pulse operation can always be achieved, given sufficient inhomogeneous gain broadening.

is comprised of inhomogeneous gain broadening, stable multi-frequency mode-locking will be achieved for all $N$. Indeed, these results are consistent with numerical simulations ${ }^{12}$ and experimental findings. ${ }^{19}$

\section{CONCLUSIONS}

The analysis presented in this manuscript considers a low-dimensional theoretical description of the mode-locked dynamics in a multiple wavelength mode of operation. Although the multi-frequency models have been considered previously, ${ }^{10-13}$ here we provide a detailed stability and bifurcation analysis of the low-dimensional reduction. We demonstrate that the stability of the multi-frequency lasing depends critically on the parameter $\alpha$ which measures the ratio of homogeneous and inhomogeneous gain broadening effects. Indeed, in order for multifrequency operation to occur and be stabilized, a sufficient amount of inhomogeneous (channel self-saturation) gain broadening must be present. Such theoretical findings are in agreement with experimental efforts in WDM systems to enhance the inhomogeneous gain broadening for stabilizing lightwave systems. ${ }^{19}$ For dual-frequency operation, the transition in mode-locking stability as a function of the parameter $\alpha$ is completely characterized by a center manifold reduction when the largest eigenvalue passes through zero. The center manifold reduction shows the fundamental bifurcation structure at the stability transition to be a supercritical pitchfork bifurcation.

This bifurcation analysis applies in the transition from single frequency operation to dual-frequency operation with differing amplitudes. It also applies to the transition from dual-frequency operation as it goes from identical to differing amplitudes. Thus the normal form reduction completely characterizes the stability transition and bifurcation structure in the three possible operating regimes of the laser. Various perturbations to the stability structure are also considered, including the effects of the cubic-quintic saturable absorption terms in the full governing equations. These terms also have a profound impact on the multi-frequency mode-locking performance and stability of the laser. Specifically, these terms can shift the region of stable two-pulse operation as a function of the parameter $\alpha$. Further, they can broaden the range of parameter space for which pulses with different amplitudes can be stabilized.

From an applications viewpoint, the low-dimensional model derived clearly demonstrates the critical interplay between homogeneous and inhomogeneous gain broadening effects. Specifically, only a small amount of inhomogeneous gain-broadening allows for multi-frequency operation in the laser. Further, bound-state (locked in time) mode-locking at multiple frequencies can be supported. Thus the mode-locking process counteracts the effects of group-velocity walk-off between neighboring frequency channels. Thus the theoretical analysis of the multi-frequency mode-locking indicates that such a mode-locking device is feasible and technologically relevant as has been demonstrated by limited experimental findings. ${ }^{3-6}$ Further, the findings validate the efforts in the WDM community to enhance the inhomogeneous gain broadening ${ }^{19}$ as this is clearly the key to producing stable 
multi-frequency operation. With the increasing demand for increased optical bandwidth, the multi-frequency mode-locking model provides a promising source for WDM signal generation which can be implemented in enabling WDM/OTDM technologies.

\section{Acknowledgements}

J. N. Kutz acknowledges support from the National Science Foundation (DMS-0604700) and the Air Force Office of Scientific Research (FA9550-09-0147).

\section{REFERENCES}

[1] G.P. Agrawal, Nonlinear Fiber Optics, Academic Press, New York, 1989.

[2] A. Hasegawa and F. Tappert, "Transmission of stationary nonlinear optical pulses in dispersive dielectric fibers. I. Anomalous dispersion," Appl. Phys. Lett. 23, 142 (1973).

[3] Y. Shiquan, L. Zhaohui, Y. Shuzhong, D. Xiaoyyi, K. Guiyun, and Z. Qida, "Dual-wavelength actively modelocked erbium dobed fiber laser using FBGs," Proceedings of SPIE Vol. 4974, 43-49 (SPIE, Bellingham, WA 2003).

[4] H. Dong, G. Zhu, Q. Wang, and N. K. Dutta, "Simultaneous mode locked operation of a fiber laser at two wavelengths," Proceedings of SPIE Vol. 5349, 117-121 (SPIE, Bellingham, WA 2004).

[5] Z. Ahned and N. Onodera, "High repetition rate optical pulse generation by frequency multiplication in actively mode-locked fiber ring lasers," Elec. Lett. 32, 455-455 (1996).

[6] C. Wu and N. K. Dutta, "High repetition-rate optical pulse generation using a rational harmonic modelocked fiber laser," IEEE J. Quant. Elec. 36, 145-150 (2000).

[7] H. Haus, "Mode-locking of lasers," IEEE J. Sel. Top. Quant. Elec. 6, 1173-1185 (2000).

[8] J. N. Kutz, "Mode-locked soliton lasers," SIAM Rev. 48, 629-678 (2006).

[9] I. N. Duling III and M. L. Dennis, Compact sources of ultrashort pulses. Cambridge, U.K.: Cambridge University Press, 1995.

[10] E. Farnum, L. Butson, and J. N. Kutz, "Theory and simulation of dual-frequency mode-locked lasers," J. Opt. Soc. Am. B 23, 257-264 (2006).

[11] E. Farnum and J. Kutz "Multifrequency mode-locked lasers" J. Opt. Soc. Am. B25,1002-1010 (2008).

[12] B. Bale, E. Farnum and J. Kutz "Dynamics of multifrequency mode-locking driven by homogeneous and inhomogeneous gain broadening effects," J. Opt. Soc. Am. B 25, 1479-1487 (2008).

[13] B. Bale, E. Farnum and J. Kutz "Theory and Simulation of Passive multi-frequency mode-locking with waveguide arrays," IEEE J. Quant. Elec. 44, 976-983 (2008)

[14] H. Leblond, M. Salhi, A. Hideur, T. Chartier, M. Brunel, and F. Sanchez, "Experimental and theoretical study of the passively mode-locked ytterbium-doped double-clad fiber laser," Phys. Rev. A 65, 063811 (2002).

[15] A. Komarov, H. Leblond, and F. Sanchez, "Quintic complex Ginzburg-Landau model for ring fiber lasers," Phys. Rev. E 72, 025604 (2005).

[16] A. Komarov, H. Leblond, and F. Sanchez, "Multistability and hysterisis phenomena in passively mode-locked fiber lasers," Phys. Rev. A 71, 053809 (2005).

[17] A. Hasegawa and Y. Kodama, Solitons in Optical Communications, (Oxford, 1995), See Chapter 10.

[18] L. F. Mollenauer, S. G. Evangelides, and J. P. Gordon, "Wavelength division multiplexing with solitons in ultra-longdistance transmission using lumped amplifiers," J. Lightwave Tech. 9, 362-367 (1991), See Appendix.

[19] E. Desurvire, Erbium-Doped Fiber Amplifiers Principles and Applications (Wiley-Interscience, New York, 1994).

[20] B. Malomed, "Variational methods in nonlinear fiber optics and related fields," Prog. Optics 43, 69-191 (2002).

[21] B. Bale, J. Kutz, "Variational Method for Mode-locking," J. Opt. Soc. Am. B, 25 1193-1202 (2008) .

[22] C. Jirauschek, U. Morgner, and F. Kärtner, "Variational analysis of spatio-temporal pulse dynamics in dispersive Kerr media," J. Opt. Soc. Am. B 19, 1716-1721 (2002). 
[23] C. Antonelli, J. Chen and F. Kärtner, "Intracavity pulse dynamics and stability for passively mode-locked lasers," Opt. Express 15, 5919-5924 (2007).

[24] N. Usechak, G. Agrawal, "Semi-analytic technique for analyzing mode-locked lasers," Opt. Express 13, 2075-2081 (2005).

[25] N. Usechak, G. Agrawal, "Rate-equation approach for frequency-modulation mode locking using the moment method," J. Opt. Soc. Am. B 22, 2570-2580 (2005).

[26] S. Namiki, E. Ippen, H. Haus, C. Yu, "Energy rate equations for mode-locked lasers," J. Opt. Soc. Am. B 14, 2099-2111 (1997).

[27] S. Namiki, E. Ippen, H. Haus, C. Yu, "Energy rate equations for mode-locked lasers," J. Opt. Soc. Am. B 14, 2099-2111 (1997).

[28] E. Tsoy, A. Ankiewicz, N. Akhmediev, "Dynamical models for dissipative localized waves of the complex Ginzburg-Landau equation," Phys. Rev. E 73, 036621 (2006).

[29] D. Anderson, M. Lisak and A. Berntson, "A variational approach to nonlinear evolution equations in optics," Pramana J. Phys. 57, 917 - 936 (2001).

[30] G. Whitham, Linear and Nonlinear Waves. Wiley, 1974.

[31] P. G. Drazin, Nonlinear Systems. Cambridge, U.K.: Cambridge University Press, 1992.

[32] J. Guckenheimer and P. Holmes, Nonlinear Oscillations, Dynamical Systems and Bifurcation of Vector Feields (Springer, 1983).

[33] S. Wiggins, Introduction To Applied Nonlinear Dynamical Systems and Chaos (Springer, 2003).

[34] T. Kapitula, J. N. Kutz, and B. Sandstede, "Stability of Pulses in the Master-Modelocking Equation," J. Opt. Soc. Am. B 19 740-746 (2002). 